\title{
Metabolic syndrome and insulin resistance in PCOS phenotypes
}

\author{
Sheena Sobti ${ }^{1 *}$, Rupali Dewan ${ }^{1}$, Sunil Ranga ${ }^{2}$ \\ ${ }^{1}$ Department of Obstetrics and Gynecology, ${ }^{2}$ Department of Pathology, Vardhman Mahavir Medical College and \\ Safdarjung Hospital, New Delhi, India
}

Received: 01 September 2017

Accepted: 25 September 2017

\section{*Correspondence:}

Dr. Sheena Sobti,

E-mail: sheena.sobti@gmail.com

Copyright: $\odot$ the author(s), publisher and licensee Medip Academy. This is an open-access article distributed under the terms of the Creative Commons Attribution Non-Commercial License, which permits unrestricted non-commercial use, distribution, and reproduction in any medium, provided the original work is properly cited.

\begin{abstract}
Background: Polycystic ovary syndrome(PCOS) is an endocrine metabolic disorder which is rapidly gaining epidemic proportions. Hyperinsulinemia and insulin resistance (IR) are thought to be key pathological factors. This study was undertaken to characterize the phenotypes of PCOS and to determine the prevalence of metabolic syndrome (MetS) and insulin resistance in them.

Methods: This observational cross-sectional study was undertaken to assess the distribution of the Rotterdam PCOS phenotypes and to report the prevalence and risk factors for MetS syndrome and insulin resistance using homeostasis model assesment for insulin resistance (HOMA-IR). 90 women aged 18-35 years newly diagnosed with PCOS were classified into one of the four potential PCOS phenotypes based on history, examination and investigations.

Results: Phenotype A was the most prevalent phenotype (45.5\%). Prevalence of insulin resistance in our study was $31 \%$ using HOMA- IR cutoff of 2.5, with highest prevalence in phenotype A and least in phenotype D. The overall prevalence of MetS was 36\% with a two- to six-fold higher prevalence in hyperandrogenic phenotypes compared to the non-hyperandrogenic phenotype. Highest mean hs- CRP was found in phenotype A which could possibly indicate greater cardiovascular risk in future. Univariate logistic regression for predictive association of MetS parameters was significantly high for deranged parameters i.e. WC $\geq 80 \mathrm{~cm}$, fasting plasma glucose $\geq 100 \mathrm{mg} / \mathrm{dl}, \mathrm{HDL} \leq 50 \mathrm{mg} / \mathrm{dl}$ and WHR $\geq 0.85$. Strong positive association was found with all these parameters $(\mathrm{p}<0.001)$ Hirsutism (modified Ferriman Gallwey score $\geq 8$ ) was strongly associated with MetS ( $\mathrm{p}=0.005$ ).

Conclusions: An appropriate diagnosis of PCOS and accurate dentification of phenotype is important as it has longterm health implications for women. We recommend screening all hyperandrogenic PCOS women for IR and metabolic abnormalities. This study has shown that HOMA-IR is a valuable tool in identifying PCOS women with metabolic syndrome and also serve to identify PCOS subtype at high risk of future metabolic syndrome.
\end{abstract}

Keywords: Insulin resistance, Metabolic syndrome, Polycystic ovary syndrome, Phenotypes

\section{INTRODUCTION}

The polycystic ovary syndrome (PCOS) is an endocrine metabolic disorder affecting $7 \%$ to $10 \%$ of women of reproductive age. ${ }^{1}$ Hyperinsulinemia and insulin resistance (IR) are thought to be key pathological factors for PCOS. The association between IR and PCOS has important clinical ramifications, particularly as IR is thought to be the uniting pathogenic factor in the associations between hypertension, glucose intolerance, obesity, lipid abnormalities and coronary artery disease, which together constitute metabolic syndrome (MetS) or syndrome ' $\mathrm{X} .{ }^{2}$ The newer phenotypes generated by the Rotterdam criteria are inadequately studied and reported. This study was undertaken to characterize the various phenotypes of PCOS, assess their distribution and to 
report the prevalence and risk factors for metabolic syndrome. The results will highlight the importance of early and regular screening in these women so that appropriate steps can be taken at the right stage to avoid full blown complications later in life.

\section{METHODS}

This observational cross-sectional study was conducted in the Department of Obstetrics and Gynecology in Safdarjung Hospital, New Delhi, after getting clearance from the Institute Ethics Committee. The study was carried out on 90 women aged 18-35 years newly diagnosed with PCOS, by Rotterdam ESHRE/ASRM PCOS group's revised 2003 criteria.

Based on history, examination and investigations, these women were then classified into one of the four potential PCOS phenotypes. ${ }^{3}$

- Phenotype A: Polycystic ovarian morphology + Hyperandrogenemia +Oligo/anovulation $(\mathrm{P}+\mathrm{H}+\mathrm{O}$, also called PCOS complete/severe PCOS)

- Phenotype B: Hyperandrogenemia +Oligo/anovulation $(\mathrm{H}+\mathrm{O})$

- Phenotype C: Polycystic ovarian morphology + Hyperandrogenemia $(\mathrm{P}+\mathrm{H}$ or ovulatory PCOS)

- Phenotype D: Polycystic ovarian morphology +Oligo/anovulation ( $\mathrm{P}+\mathrm{O}$ or mild PCOS).

Women with endocrinal disorders and on medications affecting end-points including insulin sensitizers, antiandrogens and hormonal contraceptives were excluded.

All anthropometric measurements were done following standard protocol and calibrated instruments. Waist circumference was measured in centimetres at the level of the umbilicus without clothing in standing position and hip circumference was measured at the utmost circumference with undergarment. Height was measured without shoes against a wall-fixed tape. Weight with light clothing and without shoes on a platform scale The BMI was calculated as weight/ height ${ }^{2}\left(\mathrm{~kg} / \mathrm{m}^{2}\right)$. Clinical signs of hyperandrogenism (acne, oily skin, and hirsutism) was noted. All clinical findings like Ferriman Gallwey score, galactorrhea, etc., were evaluated by single gynaecologist (author). Clinical hyperandrogenism was defined as modified Ferriman Gallway score $\geq 8$. ${ }^{4}$

Sample Collection and Estimation: Following investigations were done in fasting women $(>12 \mathrm{~h})$ recruited for study: Fasting glucose estimation, ELISA for insulin level estimation (in $\mu \mathrm{U} / \mathrm{ml}$ ), Serum Testosterone, TSH (to rule out hypothyroidism) and lipid profile. Abdominal/ transvaginal sonography was performed in early follicular phase in Safdarjung hospital radiology department. Polycystic ovary was defined as $\geq 12$ follicles per ovary and/or ovarian volume $\geq 10 \mathrm{~cm}^{3}$.
Insulin resistance was assessed using the homeostatic model assessment-insulin resistance (HOMA-IR) formula.

\section{HOMA-IR = IXG/405}

Where $\mathrm{I}=$ fasting insulin $(\mu \mathrm{U} / \mathrm{ml}), \mathrm{G}=$ fasting glucose $(\mathrm{mg} / \mathrm{dl})$. Insulin resistance was defined by HOMA-IR value of $\geq 2.5$. $^{5}$

Metabolic syndrome was assessed according to the modified AHA/NHLBI ATP III (2005) definition as the co-occurrence of three or more of the following risk factors. ${ }^{6,7}$

- Central obesity with waist circumference $\geq 80 \mathrm{~cm}$ in women,

- Elevated systolic and/or diastolic blood pressure of $\geq 130 / 85 \mathrm{mmHg}$,

- Impaired fasting serum glucose $\geq 100 \mathrm{mg} / \mathrm{dL}$,

- Elevated fasting serum triglycerides $\geq 150 \mathrm{mg} / \mathrm{dL}$, and

- Fasting high-density lipoprotein (HDL) cholesterol $<50 \mathrm{mg} / \mathrm{dL}$.

\section{Statistical analysis}

A sample size of 90 women for study was calculated assuming prevalence of PCOS as $30.44 \%$ (Kar, 2013) with absolute margin of error as $10 \%$ and $95 \%$ confidence limit. The prevalence of IR and MetS in each phenotype was compared. Statistical analysis was performed by the SPSS program for Windows, version 17.0. Continuous variables are presented as mean $\pm S D$, and categorical variables are presented as absolute numbers and percentage. Data were checked for normality before statistical analysis using Shaipro Wilk test. Normally distributed continuous variables were compared using ANOVA. If the F value was significant and variance was homogeneous, Tukey multiple comparison test was used to assess the differences between the individual groups; otherwise, Tamhane's T2 test was used. Categorical variables were analyzed using the chi square test. Post hoc analysis, univariate logistic analysis and $\mathrm{T}$ test was used to find predictive association of variables. For all statistical tests, a $\mathrm{p}$ value less than 0.05 was taken to indicate a significant difference.

\section{RESULTS}

Out of 90 women, forty-one women (45.5\%) met all 3 Rotterdam criteria (PCOS complete or phenotype A). This was the most prevalent phenotype (Fig. 1). The second most common phenotype was mild PCOS or phenotype D (23.4\%). The least common phenotype was phenotype B (14.4\%)

The mean and SD of clinical and biochemical profile of these four phenotypes are presented in Table 1. 
Table 1: Clinical and biochemical profile of PCOS phenotypes.

\begin{tabular}{|llllll|}
\hline Characteristic & Phenotype A & Phenotype B & Phenotype C & Phenotype D & P value \\
\hline Age $($ years $)$ & $\mathrm{N}=41$ & $\mathrm{~N}=13$ & $\mathrm{~N}=15$ & $\mathrm{~N}=21$ & \\
\hline BMI $\left(\mathrm{kg} / \mathrm{m}^{2}\right)$ & $26.39 \pm 2.65$ & $26.46 \pm 3.38$ & $25.87 \pm 2.56$ & $25.00 \pm 2.49$ & 0.258 \\
\hline WHR & $23.22 \pm 3.05$ & $22.69 \pm 2.90$ & $23.87 \pm 2.88$ & $22.76 \pm 2.95$ & 0.670 \\
\hline WC $(\mathrm{cm})$ & $0.84 \pm 0.04$ & $0.82 \pm 0.03$ & $0.83 \pm 0.03$ & $0.81 \pm 0.03$ & 0.008 \\
\hline FG Score & $75.37 \pm 8.90$ & $77.00 \pm 7.10$ & $77.20 \pm 6.21$ & $75.71 \pm 5.98$ & 0.825 \\
\hline FBG $(\mathrm{mg} / \mathrm{dl})$ & $11.27 \pm 1.67$ & $11.15 \pm 1.82$ & $11.33 \pm 1.63$ & $3.90 \pm 1.38$ & $<0.001$ \\
\hline FI $(\mu \mathrm{U} / \mathrm{dl})$ & $95.49 \pm 7.40$ & $92.38 \pm 8.66$ & $89.33 \pm 7.40$ & $87.62 \pm 7.55$ & 0.001 \\
\hline HOMA-IR & $14.81 \pm 6.95$ & $12.50 \pm 5.96$ & $11.23 \pm 4.90$ & $9.56 \pm 2.71$ & 0.007 \\
\hline HDL- C $(\mathrm{mg} / \mathrm{dl})$ & $3.61 \pm 1.90$ & $2.96 \pm 1.66$ & $2.47 \pm 1.30$ & $1.97 \pm 0.47$ & 0.001 \\
\hline FTG $(\mathrm{mg} / \mathrm{dl})$ & $136.10 \pm 9.09$ & $44.92 \pm 6.08$ & $47.00 \pm 5.26$ & $49.05 \pm 2.40$ & 0.020 \\
\hline Testosterone $(\mathrm{ng} / \mathrm{dl})$ & $0.873 \pm 0.383$ & $131.62 \pm 7.32$ & $131.00 \pm 6.06$ & $131.38 \pm 6.95$ & 0.069 \\
\hline
\end{tabular}

Out of 90 women, 75 women $(83.3 \%)$ presented with irregular menses. This was the most common complaint. The second most common complaint was excessive hair growth $(43.3 \%$ of study cohort) followed by acne $(31.1 \%)$.

Among the study cohort, 19 out of the 30 married women presented with inability to conceive (primary infertility), making incidence of primary infertility $63.3 \%$ in these women. None of the study subjects was found to be hypertensive.

Overall prevalence of obesity was $48.9 \%$ (according to consensus statement for Asian Indian) and the frequency of obese women was maximum in phenotype $\mathrm{C}(\mathrm{H}+\mathrm{O}$, $60 \%)$ followed by phenotype $\mathrm{A}(\mathrm{P}+\mathrm{H}+\mathrm{O}, 51.2 \%){ }^{8}$

Waist hip ratio (WHR) equal to or greater than 0.85 (central obesity) was found in $26.7 \%$ of the study cohort (24 out of 90 women). Central obesity was found in nearly one-third of women in phenotypes $\mathrm{A}$ and $\mathrm{C}$ (36.6\% and $33.3 \%$ respectively. Least prevalence of central obesity was found in phenotype D.

Modified Ferriman- Gallwey (mFG) score showed a gradient among the phenotypes, being highest in phenotype $\mathrm{C}(\mathrm{H}+\mathrm{P}, 11.33 \pm 1.63)$ followed by phenotype A $(\mathrm{P}+\mathrm{H}+\mathrm{O}, 11.27 \pm 1.67)$ and phenotype $\mathrm{B}(\mathrm{H}+\mathrm{O}$, $11.15 \pm 1.82)$ and least in phenotype $\mathrm{D}(3.90 \pm 1.38)$.

Insulin resistance (HOMA-IR $\geq 2.5$ ) was present in $31 \%$ of all PCOS women. Prevalence of IR varied in different phenotypes. It was highest in phenotype A i.e PCOS complete $(48 \%)$ followed by phenotype B. Least prevalence was found in phenotype D (14\%).

BMI, waist circumference and WHR were found to be positively correlated with HOMA-IR i.e. insulin resistance. Fasting HDL was negatively correlated with HOMA-IR. No correlation could be found with hypertension as all our study subjects were normotensive (Table 2).

Table 2: Predictive association of various parameters with HOMA-IR.

\begin{tabular}{|lll|}
\hline Parameter & $\begin{array}{l}\text { HOMA-IR } \\
\text { Correlation } \\
\text { coefficient }(\mathbf{r})\end{array}$ & p value \\
\hline Age $($ years $)$ & 0.203 & 0.055 \\
\hline Height $(\mathrm{cm})$ & -0.074 & 0.491 \\
\hline Weight $(\mathrm{kg})$ & $0.549 * *$ & $<0.001$ \\
\hline BMI $(\mathrm{kg} / \mathrm{m} 2)$ & $0.586^{* *}$ & $<0.001$ \\
\hline WC $(\mathrm{cm})$ & $0.623^{* *}$ & $<0.001$ \\
\hline WHR & $0.534^{* *}$ & $<0.001$ \\
\hline HC $(\mathrm{cm})$ & $0.492^{* *}$ & $<0.001$ \\
\hline FPG $(\mathrm{mg} / \mathrm{dl})$ & $0.695^{* *}$ & $<0.001$ \\
\hline FI $(\mu \mathrm{U} / \mathrm{ml})$ & $0.974^{* *}$ & $<0.001$ \\
\hline Testosterone $(\mathrm{ng} / \mathrm{ml})$ & 0.092 & 0.39 \\
\hline FG score & $0.252^{*}$ & 0.017 \\
\hline hsCRP $(\mathrm{mg} / \mathrm{l})$ & 0.091 & 0.395 \\
\hline F.TG $(\mathrm{mg} \%)$ & $0.240^{*}$ & 0.023 \\
\hline F.HDL $(\mathrm{mg} \%)$ & $-0.600^{* *}$ & $<0.001$ \\
\hline
\end{tabular}

HOMA-IR cut-off of 2.5 in present study showed $90 \%$ sensitivity and $98.3 \%$ specificity for predicting metabolic syndrome in PCOS. Positive predictive value is $96.4 \%$ (Table 3).

Table 3: Prevalence of insulin resistance in PCOS women with metabolic syndrome.

\begin{tabular}{|lll|l|}
\hline $\begin{array}{l}\text { Insulin } \\
\text { resistance }\end{array}$ & $\begin{array}{l}\text { PCOS women } \\
\text { with MS }\end{array}$ & $\begin{array}{l}\text { PCOS women } \\
\text { without MS }\end{array}$ & Total \\
\hline $\begin{array}{l}\text { HOMA- } \\
\text { IR } \geq 2.5\end{array}$ & 27 & 1 & 28 \\
\hline $\begin{array}{l}\text { HOMA- } \\
\text { IR }<2.5\end{array}$ & 3 & 59 & 62 \\
\hline Total & 30 & 60 & 90 \\
\hline
\end{tabular}


The overall prevalence of metabolic syndrome in PCOS women in present study was $36 \%$. Highest prevalence of metabolic syndrome was found in the PCO complete phenotype A (23 out of 41, 56\%). Prevalence of metabolic syndrome in phenotypes $\mathrm{B}$ and $\mathrm{C}$ is $38.5 \%$ ( 5 out of 13) and $20 \%$ (3 out of 15) respectively. In phenotype $\mathrm{D}$ the prevalence of metabolic syndrome was lowest $(9 \%)$. This is statistically significant $(\mathrm{p}=0.002)$. Univariate logistic regression showing predictive association of metabolic syndrome parameters in different phenotypes of PCOS was significantly high for deranged parameters i.e. $\mathrm{WC} \geq 80 \mathrm{~cm}$, fasting plasma glucose $\geq 100 \mathrm{mg} / \mathrm{dl}, \mathrm{HDL} \leq 50 \mathrm{mg} / \mathrm{dl}$ and WHR $\geq 0.85$. Strong positive association was found with all these parameters $(\mathrm{p}<0.001)$. No correlation could be found with hypertension as all our study subjects were normotensive.

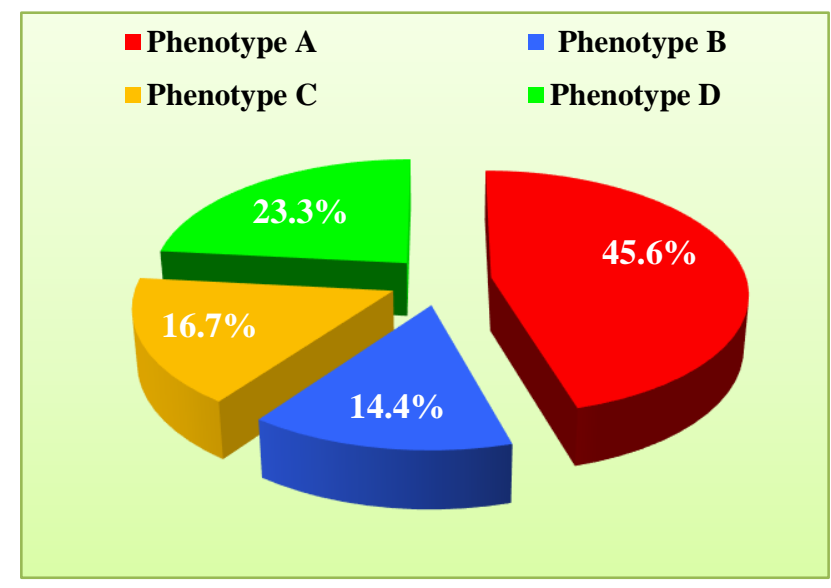

Figure 1: Distribution of PCOS phenotypes.

Hirsutism (modified Ferriman Gallwey score $\geq 8$ ) was significantly associated with metabolic syndrome. Also, obesity i.e. $\mathrm{BMI} \geq 25$ and $\mathrm{WC} \geq 80 \mathrm{~cm}$ showed strong association with metabolic syndrome.

\section{DISCUSSION}

Prevalence of women with PCOS was maximum for phenotype $\mathrm{A}(\mathrm{H}+\mathrm{P}+\mathrm{O} ; 45.5 \%)$ followed by phenotype $\mathrm{D}$ $(\mathrm{O}+\mathrm{P} ; 23.4 \%)$, phenotype $\mathrm{C}(\mathrm{H}+\mathrm{P} ; 16.7 \%)$ and phenotype $\mathrm{B}(\mathrm{H}+\mathrm{O} ; 14.4 \%)$. Nearly $3 / 4^{\text {th }}$ of $\mathrm{PCOS}$ women belonged to hyperandrogenic phenotypes with phenotype A being most prevalent. Normoandrogenic phenotype $(\mathrm{P}+\mathrm{O})$ comprised a significant proportion of PCOS women, because these women are often symptomatic with irregular cycles and characteristic findings on ultrasongraphy. Ovulatory phenotype $\mathrm{P}+\mathrm{H}$, and phenotype $\mathrm{B}(\mathrm{H}+\mathrm{O})$ was the least common group, possibly because these women are mostly asymptomatic and unlikely to present in gynecology outpatient. Also, women with hyperandrogenic symptoms like acne and hirsutism are more likely to consult dermatology.

In contrast to present study, Mehrabian et al in Turkey reported phenotype D as the most prevalent phenotype. ${ }^{9}$ A study by Joshi et al among adolescent and young girls in Mumbai also found mild PCOS (phenotype D) to be most common $(52.6 \%) .{ }^{10}$ One of the reasons for the higher prevalence of phenotype $\mathrm{D}$ in their study can be the lower age of the study cohort (it was conducted among adolescents and young girls aged 15-24 years). Our study also observed that PCOS women belonging to phenotype D presented at younger mean age group with $61 \%$ women aged less than 25 years. These girls were brought by their parents in view of irregular menstrual cycles and their concern regarding its effect on future reproductive outcomes. Saxena et al reported phenotype $\mathrm{C}$ as most common. Their study subjects were infertile women presenting in a tertiary care centre. ${ }^{11}$ The difference in prevalence of different phenotypes can be attributed to the genetic, racial/ethnic and geographic variations. Phenotype may also vary depending upon the study population from where patients were recruited.

Oligo/ anovulation leading to irregular menses is one of the diagnostic criteria for PCOS. This was the most common presenting complaint of the women in our study. Irregular menses accounted for $83 \%$ of study cohort. Menstrual irregularities are seen in all phenotypes of PCS except phenotype C. A study by Ramanand et al in Maharashtra reported oligomenorrheoa in 65\% PCOS cases and recommend oligomenorrheoa as a highly predictive surrogate marker of PCOS. ${ }^{12}$ Nurses' Health study II reported that over an 8-year period, the conversion rate to type 2 diabetes among oligomenorrheic women was approximately two-fold greater than that for eumenorrheic women, regardless of whether the oligomenorrheic women were obese or lean, indicating that oligomenorrhea was an independent predictor of type 2 diabetes. ${ }^{13}$ On the background of this association the high percentage of irregular menses in the present study warrants a serious thought toward this menstrual problem.

Majority of women $(66.7 \%)$ were unmarried. However, 19 out of 30 married women $(63.3 \%)$ in study cohort had primary infertility. Gorry et al reported that polycystic ovary syndrome accounts for more than $75 \%$ of cases of anovulatory infertility. The mechanism of anovulation is uncertain but there is evidence that arrested antral follicle development is associated with the abnormal endocrine profile, in particular the interaction of insulin and LH on granulosa cell differentiation. ${ }^{14}$

In present study, $43.3 \%$ (39 out of 90) women presented with complaint of excessive hair growth and $31.1 \%$ complained of acne. In some women, acne and hirsutism were present independently. Overall $85.6 \%$ of women in our study had hyperandrogenic PCOS (phenotypes A, B and C).

The modified Ferriman-Gallwey score (mfG) of 8 and above over 9 body parts defines hirsutism. Highest $\mathrm{mfG}$ score was seen in phenotype $\mathrm{C}$ and least in phenotype $\mathrm{D}$. This was found to be statistically significant $(\mathrm{P}<0.001)$. 
In present study, there was no statistically significant difference in serum testosterone in the 4 phenotypes. This is supported by the study by Ramanand et al in which serum testosterone levels were either normal or low in most patients. $^{12}$ In PCOS, MS risk increases with increasing androgen levels, independent of insulin resistance and obesity, and antagonizing androgen action ameliorates features of MS. ${ }^{15}$ In this context the high prevalence of hirsutism and acne in the present study necessitates suitable measures towards this problem.

Nearly half of the women in our study were obese. Contrary to our study, $62.5 \%$ of PCOS women were found to be overweight by Kar. ${ }^{16}$ The number of obese and overweight women was also higher $(75 \%)$ in the study by Ramanand et al which was conducted in an endocrinology hospital which could have influenced the selection of patients. ${ }^{12}$ It is also a possibility that obese women, because of obesity become more health conscious, and/or have co-morbidities and seek medical advice as compared to non-obese women.

Waist circumference $\geq 80 \mathrm{~cm}$ is one of the diagnostic criteria for metabolic syndrome and was found in $37.8 \%$ women in our study cohort. In phenotype A, nearly half of the women had waist circumference more than $80 \mathrm{~cm}$, compared to only one-fifth of the women in phenotype D. Waist hip ratio equal to or greater than 0.85 is indicative of central obesity, and was found in $26.7 \%$ of PCOS women overall. Central obesity was found in nearly onethird of women in phenotypes $\mathrm{A}$ and $\mathrm{C}$ (36.6\% and $33.3 \%$ respectively). Least prevalence of central obesity was found in phenotype D. Kar also observed that a significant proportion of lean women had abnormal WHR. In this study, android central obesity was found to be correlated with risk of metabolic syndrome. ${ }^{16}$ As android obesity is a known risk factor in development of cardiovascular diseases in future, this finding is of concern and warrants lifestyle modification and regular follow up of metabolic parameters even in lean PCOS women.

In present study, insulin resistance has been reported based on HOMA-IR values. Diagnosis of IR is not standardized. The gold standard for establishing IR is euglycemic hyperinsulinemic clamp. However, this elaborate procedure is not suitable as a screening method. HOMA-IR calculation correlates very well with euglycemic hyperinsulinemic clamp and is often used as a surrogate marker. However, the cut-off or threshold values for HOMA-IR are not established. True prevalence is difficult to determine with no universally accepted parameter to measure IR. Currently there is lack of consensus on how best to evaluate insulin sensitivity. Homeostatis measurements (fasting glucose/insulin ratio or HOMA-IR values) and minimal model tests (OGTT) represent the easiest screening methods. ${ }^{17}$

Insulin resistance (HOMA-IR $\geq 2.5$ ) was present in $31.1 \%$ of all PCOS women. Prevalence of IR varied in different phenotypes. It was highest in phenotype A i.e. PCOS complete $(48 \%)$ followed by phenotype B. Least prevalence was found in phenotype D (14\%). This was found to be statistically significant $(p=0.049$. This is similar to the findings of Kar who reported the prevalence of insulin resistance to be $30.44 \%$. In our study, highest prevalence of insulin resistance was found in phenotype A and least in phenotype D. This was statistically significant $(\mathrm{p}=0.002)$.

Present study observed BMI, waist circumference and WHR to be positively associated with HOMA-IR i.e. insulin resistance. Fasting HDL was negatively correlated with HOMA-IR. No correlation could be found with hypertension as all our study subjects were normotensive.

Prevalence of metabolic syndrome was found to be $36 \%$. Present study found that the hyperandrogenic phenotypes (phenotypes A, B and C) had a two- to six-fold higher (20-56\%) prevalence of metabolic syndrome compared to phenotype D (9\%), suggesting that the nonhyperandrogenic phenotype has a mild metabolic profile. Shroff et al had also observed a similar and significantly higher risk of MS (35- 44\%) in the three PCOS phenotypes than controls, except for the nonhyperandrogenic PCOS phenotype (OA + PCOM, $20 \%) .^{18}$

Univariate logistic regression showing predictive association of metabolic syndrome parameters in different phenotypes of PCOS was significantly high for deranged parameters i.e. $\mathrm{WC} \geq 80 \mathrm{~cm}$, fasting plasma glucose $\geq 100 \mathrm{mg} / \mathrm{dl}$, HDL $\leq 50 \mathrm{mg} / \mathrm{dl}$ and WHR $\geq 0.85$. Strong positive association was found with all these parameters $(\mathrm{p}<0.001)$. Thus, present study is in concordance with Saxena et al who reported strong positive association of metabolic syndrome with waist circumference, HDL level and fasting glucose levels. ${ }^{[12]}$ However, no correlation could be found with hypertension in our study as all our study subjects were normotensive.

Elevation of high sensitivity CRP (hs-CRP) is considered to be one of the most important predictors of the risk of cardiovascular events; the predictive power of hs-CRP is independent of and complementary to lipid profile. ${ }^{19}$ In the present study, hsCRP levels were divided into three groups <1, 1-3 and >3 mg/l corresponding to low, moderate and high risk groups for future $\mathrm{CV}$ events. In present study cohort, 92.2\% women had low risk and $7.8 \%$ had intermediate risk. No subject was found to have high risk. Highest mean hs- CRP was found in phenotype A and least in phenotype D. this could possibly indicate greater cardiovascular risk in phenotype A compared to phenotype $\mathrm{D}$ in future.

In this study, hirsutism (modified Ferriman Gallwey score $\geq 8$ ) was strongly associated with metabolic syndrome $(\mathrm{p}=0.005)$. This finding has also been found in other studies that in addition to insulin, the main 
pathophysiologic actor of MS, an abnormal sex steroid milieu also has a potential contributing role. In particular, within the context of PCOS, androgen excess may serve as an endocrine modulator of MS. Thus, in the shared pathophysiologic spectrum of PCOS and MS, androgen excess possibly acts as a triggering factor in the development of MS, playing another key role beyond the one involved in ovulatory dysfunction. This role may pertain not only to systemic but also to local tissue androgen excess. More specifically, hyperandrogenism may amplify the adverse metabolic phenotype of PCOS in a dual mode, through the aggravation of central adiposity and the perpetuation of insulin resistance.

On comparing clinical and biochemical parameters of PCOS women with and without metabolic syndrome (Table 30), there was statistically significant difference between the two groups in $\mathrm{BMI}(\mathrm{P}=0.000)$, WC $(\mathrm{P}=$ $0.000)$, waist-hip ratio $(\mathrm{P}=0.000)$, serum fasting glucose $(\mathrm{P}=0.000)$, fasting insulin $(\mathrm{p}=0.001)$, modified Ferriman Gallwey score $(\mathrm{p}=0.001)$, HDL $(\mathrm{P}=0.00)$, triglycerides $(\mathrm{P}=0.001)$ and HOMA- IR $(\mathrm{P}=0.000)$

Identification of specific clinical patterns among women with PCOS has diagnostic implications in the search for specific genetic determinants and the development of appropriate treatment regimens for various disease manifestations. The major limitation of our study is the absence of age and BMI matched control group due to nonavailability of educated and informed volunteers.

\section{CONCLUSION}

The findings of our study strongly suggest that a thorough understanding of PCOS pathophysiology and its association with reproductive and metabolic disturbances is essential for any physician addressing women's health. An appropriate diagnosis of PCOS and accurate identification of phenotype is very important as it has long-term health implications for women. These women need to be informed and counselled about their present and long-term risks. Therefore, we recommend screening all hyperandrogenic PCOS women for IR and metabolic abnormalities. This study has shown that HOMA-IR is a valuable tool in identifying PCOS women with MetS and may also serve to identify PCOS at high risk of future MS and who are amenable to early intervention.

Funding: No funding sources

Conflict of interest: None declared

Ethical approval: The study was approved by the Institutional Ethics Committee

\section{REFERENCES}

1. Neves EM, Fonseca AM, Bagnoli VR, Souza MA, Araújo Moraes SDT, Maciel GAR et al. Polycystic Ovary Syndrome: Correlation between Phenotypes and Metabolic Syndrome. J Steroids Hormon Sci. 2014;5:132.
2. Apridonidze T, Essah PA, Iuorno MJ, Nestler JE. Prevalence and characteristics of the metabolic syndrome in women with polycystic ovary syndrome. J Clin Endocrinol Metab. 2005;90:192935.

3. Carmina E. Metabolic syndrome in polycystic ovary syndrome. Minerva Ginecologica. 2006;58:109-114.

4. Yildiz BO, Bolour S, Woods K, Moore A, Azziz R. Visually scoring hirsutism. Human Reprod Update. 2010;16(1):51-64.

5. Singh Y, Garg MK, Tandon N, Marwaha RK. A study of insulin resistance by HOMA-IR and its cutoff value to identify metabolic syndrome in urban Indian adolescents. J Clin Res Pediatr Endocrinol. 2013;5:245-51.

6. Lepor NE, Vogel RE. National Cholesterol Education Program Adult Treatment Panel III. Summary of the third report of the National Cholesterol Education Program Adult Treatment Panel III. Rev Cardiovas Med. 2001;2:160-5.

7. Grundy SM, Cleeman JL, Daniels SR, Donato KA, Eckel RH, Franklin BA et al. American Heart Association, National Heart, Lung, and Blood Institute. Diagnosis and management of the metabolic syndrome: An American Heart Association/National Herat, Lung and Blood Institute Scientific Statement. Circulation. 2005;112:2735-52.

8. Misra A, Chowbey P, Makkar BM, Vikram NK, Wasir JS, Chadha D et al. Consensus statement for diagnosis of obesity, abdominal obesity and the metabolic syndrome for Asian Indians and recommendations for physical activity, medical and surgical management. J Assoc Physicians India. 2009 Feb; 57:163-70.

9. Mehrabian F, Khani B, Kelishadi R, Kermani N. The prevalence of metabolic syndrome and insulin resistance according to the phenotypic subgroups of polycystic ovary syndrome in a representative sample of Iranian females. J Res Med Sci. 2011 Jun;16(6):763-9.

10. Joshi B, Mukherjee S, Patil A, Purandare A, Chauhan S, Vaidya R. A cross-sectional study of polycystic ovarian syndrome among adolescent and young girls in Mumbai, India Indian $\mathbf{J}$ Endocrinol Metab. 2014;18:317-324.

11. Saxena P, Singh S, Bhattacharjee J. Endocrine and metabolic profile of different phenotypes of polycystic ovarian syndrome. J Obstet Gynecol India. 2016 ; 66:560-6.

12. Ramanand SJ, Ghongane BB, Ramanand JB, Patwardhan MH, Ghanghas RR, Jain SS. Clinical characteristics of polycystic ovary syndrome in Indian women. Indian J Endocr Metab. 2013;17:138145 .

13. Solomon CG, Hu FB, Dunaif A, Rich-Edwards J, Willett WC, Hunter DJ et al. Long or highly irregular menstrual cycles as a marker for risk of type 2 diabetes mellitus. JAMA. 2001;286:2421-6. 
14. Gorry A, White DM, Franks S. Infertility in polycystic ovary syndrome: focus on low-dose gonadotropin treatment. Endocrine. 2006;30:27-33.

15. Coviello AD, Legro RS, Dunaif A. Adolescent girls with polycystic ovary syndrome have an increased risk of the metabolic syndrome associated with increasing androgen levels independent of obesity and insulin resistance. J Clin Endocrinol Metab. 2006;91(2):492-7.

16. Kar S. Anthropometric, clinical, and metabolic comparisons of the four Rotterdam PCOS phenotypes: a prospective study of PCOS women. J Hum Reprod Sci. 2013;6:194-200.

17. Misra A, Vikram NK, Arya S, Pandey RM, Dhingra $\mathrm{V}$, Chatterjee A et al. High prevalence of insulin resistance in postpubertal Asian Indian children is associated with adverse truncal body fat patterning, abdominal adiposity and excess body fat. Int J Obes Relat Metab Disord. 2004;28:1217-26.

18. Shroff R, Syrop CH, Davis W, Van Voorhis BJ, Dokras A. Risk of metabolic complications in the new PCOS phenotypes based on the Rotterdam criteria. Fertil Steril. 2007;88:1389-95.

19. Ridker PM, Buring JE, Cook NR, Rifai N. C-reactive protein, the metabolic syndrome, and risk of incident cardiovascular events: an 8-year follow-up of 14719 initially healthy American women. Circulation. 2003;107:391-7.

Cite this article as: Sobti S, Dewan R, Ranga S. Metabolic syndrome and insulin resistance in PCOS phenotypes. Int J Reprod Contracept Obstet Gynecol 2017;6:5067-73. 